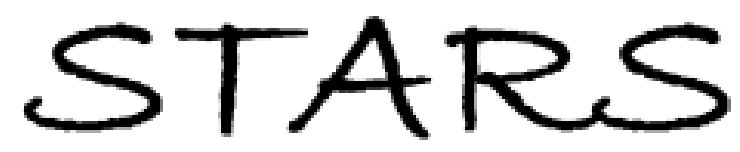

University of Central Florida

STARS

$1-1-2005$

\title{
Mega three-photon absorption cross-section enhancement in pseudoisocyanine J-aggregates
}

Ion Cohanoschi

University of Central Florida

Amel Barbot

Kevin D. Belfield

University of Central Florida

Sheng Yao

University of Central Florida

Florencio E. Hernandez

University of Central Florida

Find similar works at: https://stars.library.ucf.edu/facultybib2000

University of Central Florida Libraries http://library.ucf.edu

This Article is brought to you for free and open access by the Faculty Bibliography at STARS. It has been accepted for inclusion in Faculty Bibliography 2000s by an authorized administrator of STARS. For more information, please contactSTARS@ucf.edu.

\section{Recommended Citation}

Cohanoschi, Ion; Barbot, Amel; Belfield, Kevin D.; Yao, Sheng; and Hernandez, Florencio E., "Mega threephoton absorption cross-section enhancement in pseudoisocyanine J-aggregates" (2005). Faculty Bibliography 2000s. 5075.

https://stars.library.ucf.edu/facultybib2000/5075

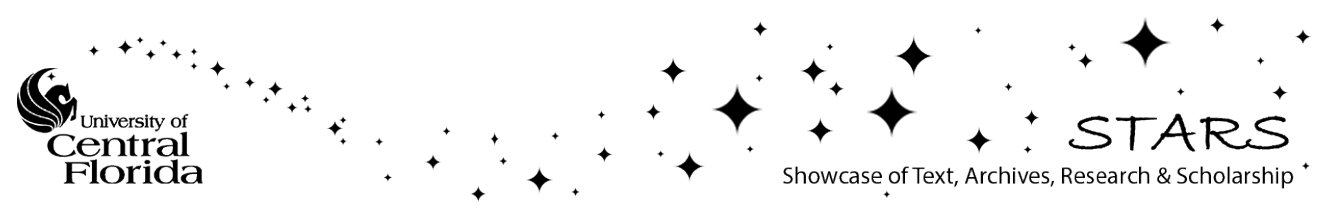




\section{Mega three-photon absorption cross-section enhancement in pseudoisocyanine $\mathrm{J}$ - aggregates}

Cite as: J. Chem. Phys. 123, 231104 (2005); https://doi.org/10.1063/1.2150209

Submitted: 04 October 2005. Accepted: 15 November 2005. Published Online: 21 December 2005

Ion Cohanoschi, Amel Barbot, Kevin D. Belfield, Sheng Yao, and Florencio E. Hernandez

\section{ARTICLES YOU MAY BE INTERESTED IN}

Density functional response theory calculations of three-photon absorption

The Journal of Chemical Physics 121, 9239 (2004); https://doi.org/10.1063/1.1804175

The dynamics of one-dimensional excitons in liquids

The Journal of Chemical Physics 102, 20 (1995); https://doi.org/10.1063/1.469393

The impact of the $\pi$-electron conjugation length on the three-photon absorption cross section of fluorene derivatives

The Journal of Chemical Physics 124, 194707 (2006); https://doi.org/10.1063/1.2202322
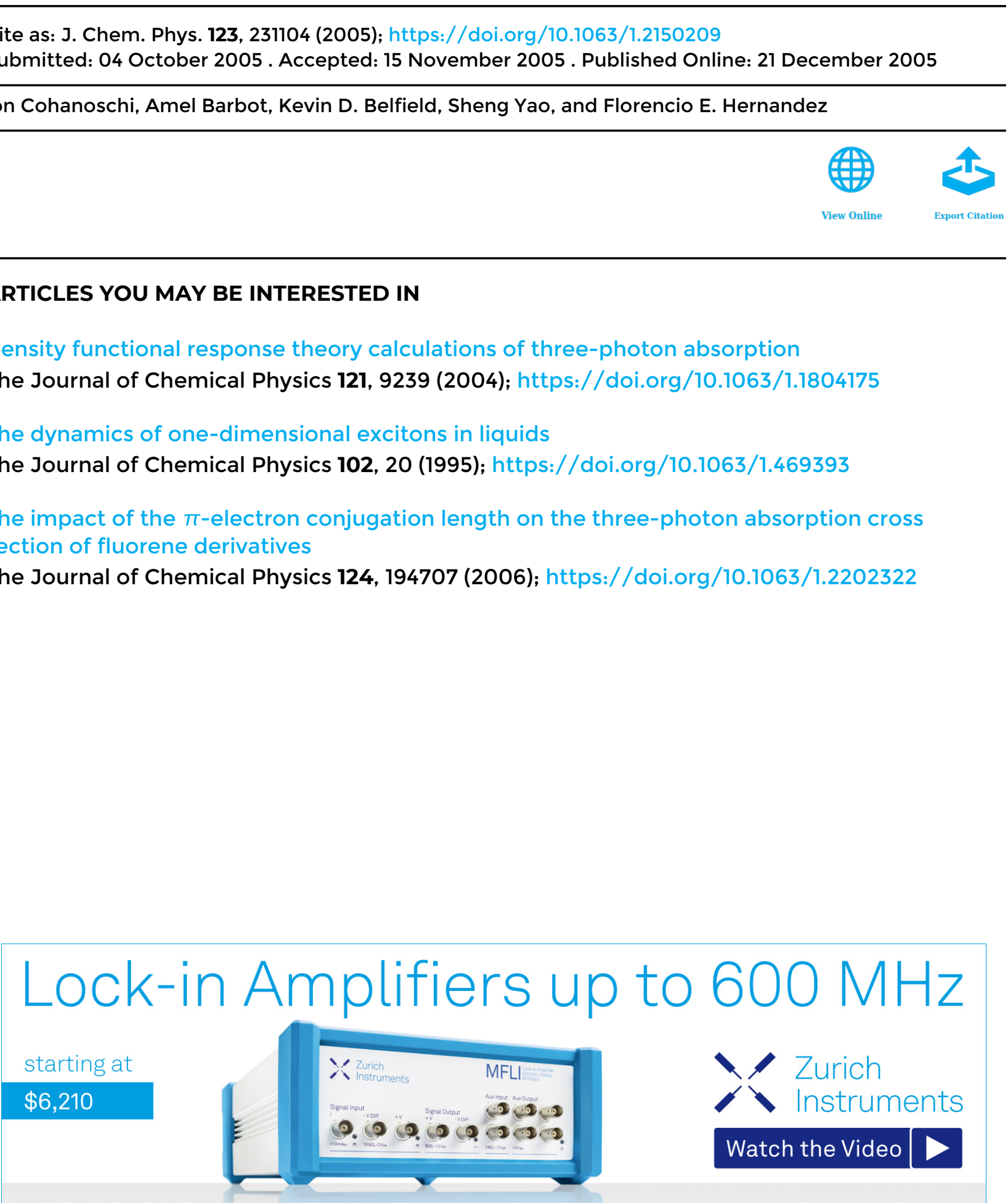

J. Chem. Phys. 123, 231104 (2005); https://doi.org/10.1063/1.2150209

123, 231104

(c) 2005 American Institute of Physics. 


\title{
Mega three-photon absorption cross-section enhancement in pseudoisocyanine J-aggregates
}

\author{
Ion Cohanoschi \\ Department of Chemistry and College of Optics and Photonics, CREOL \& FPCE, University of Central \\ Florida, P. O. Box 162366, Orlando, Florida 32816-2366 \\ Amel Barbot \\ Institut d'Optique, Centre Scientifiqe-Bâtiment 503, 91403 Orsay Cedex, France \\ Kevin D. Belfield, Sheng Yao, and Florencio E. Hernandez ${ }^{a}$ \\ Department of Chemistry and College of Optics and Photonics, CREOL \& FPCE, University of Central \\ Florida, P. O. Box 162366, Orlando, Florida 32816-2366
}

(Received 4 October 2005; accepted 15 November 2005; published online 21 December 2005)

\begin{abstract}
Herein we report an extraordinary three-photon absorption cross-section $\left(\sigma_{3}^{\prime}\right)$ enhancement in J-aggregates supramolecular systems. The much higher value of $\sigma_{3}^{\prime}$ in PIC J-aggregate $(2.5$ $\left.\times 10^{-71} \mathrm{~cm}^{6} \mathrm{~s}^{2} \mathrm{ph}^{-2}\right)$ compared to typical values obtained in organic molecules $\left(10^{-80} \mathrm{~cm}^{6} \mathrm{~s}^{2} \mathrm{ph}^{-2}\right)$ is attributed to the strong molecular transition dipole moment coupling in the supramolecular assembly. Three-photon absorption of PIC J-aggregates and monomer aqueous solutions were measured using the well known open aperture $Z$-scan technique pumping with a 25 ps pulse laser-OPG system at $1720 \mathrm{~nm}$. This novel result opens new expectations for applications of supramolecular systems in bioimaging and medicine. (c) 2005 American Institute of Physics.
\end{abstract}

[DOI: $10.1063 / 1.2150209]$

\section{BACKGROUND}

The development of new organic molecules with high three-photon absorption (3PA) has become a field of great interest in the last five years due to their potential applications in bioimaging and photodynamic therapy. ${ }^{1,2}$ Their applicability is based on the improvement in penetration depth when working in the living organism transparency windows in the near-infrared region $(1100$ to $1300 \mathrm{~nm}),{ }^{3}$ the minimization of losses due to scattering when pumping at longer wavelengths, ${ }^{4}$ and the improvement in spatial resolution. ${ }^{5}$

Three-photon absorption refers to the simultaneous absorption of three-photons in a single event through virtual states. ${ }^{6}$ 3PA in centrosymmetric molecules is subject to similar constraints imposed by quantum mechanic selection rules to single-photon absorption (1PA), i.e., 3PA gains access to a state with opposite symmetry with respect to the ground state. $^{7}$ Therefore, an enhancement in 1PA should also signify an enhancement in 3PA. Since the rate of energy absorption for a 3PA process has a cubic dependence with the irradiance of the incident radiation and, the 3PA cross-section $\sigma_{3}^{\prime}$ for organic molecules is typically low, ${ }^{8}$ high irradiances $\left(>10 \mathrm{GW} / \mathrm{cm}^{2}\right)$ are required to induce three-photon excitation. New strategies to understand the structure-property relationship for three-photon absorbing materials have been recently developed to increase $\sigma_{3}^{\prime}$ in conjugated organic systems, ${ }^{7,8}$ and new methods have been developed to enhance the electric-field intensity via surface plasmon reso-

\footnotetext{
${ }^{a)}$ Author to whom correspondence should be addressed.
}

nance to facilitate the 3PA. ${ }^{9}$ However, novel channels to further enhance $\sigma_{3}^{\prime}$ are still necessary for more practical applications.

Supramolecular assembly such as J-aggregates ${ }^{10,11}$ opens many possibilities in the field of nonlinear optics. ${ }^{12,13}$ The formation of such supramolecular aggregates has been observed at relatively high chromophore concentrations and are characterized by a sharp absorption band that is redshifted relative to the monomer. ${ }^{10,11}$ The unusual sharpness of the $J$-band is the result of the collective effects in the optical response that are caused by the strong interaction between the molecules within the aggregate. ${ }^{14}$ On the other hand, the delocalization of the electronic states over many molecules result in so called exciton states. These states can be envisaged as a coherent movement of an excitation over the chain of molecules that can result in unprecedented optical nonlinearities in J-aggregates. ${ }^{15}$ Theoretical predictions and experimental evidence of strong two-photon absorption (2PA) in J-aggregates have been reported in the literature. ${ }^{16,17}$ However, heretofore, there is no experimental evidence of higher order nonlinear absorption in supramolecular systems.

In this letter, we report for the first time a very large 3PA optical cross-section in 1,1'-diethyl-2,2'-cyanine iodide (better known as pseudoisocyanine, PIC) J-aggregate assembly. Pumping at 3 times the PIC $J$-band linear absorption $(573 \mathrm{~nm})$ in aqueous solution we show that $\sigma_{3}^{\prime}$ for PIC $\mathrm{J}$-aggregate is $\sim 10^{6}$ fold greater than typical organic molecules. No evidence of 3PA of the monomer was obtained at the same wavelength.

In Fig. 1 we show the molecular structure of PIC, and its linear absorption spectrum from 200 to $1800 \mathrm{~nm}$ for the monomer and the J-aggregate assembly, in aqueous solution, 


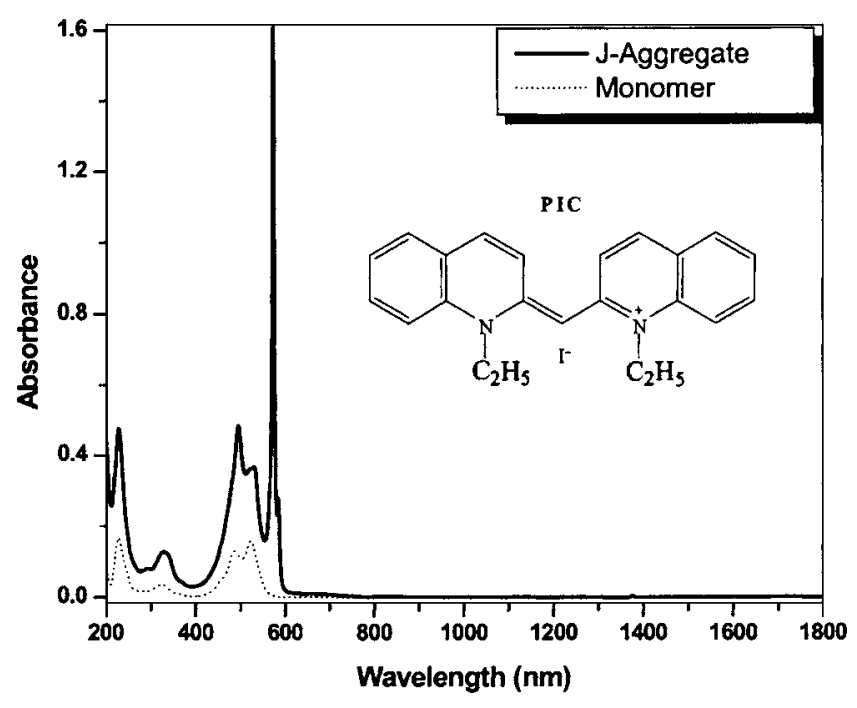

FIG. 1. Molecular structure of 1, $1^{\prime}$-diethyl-2,2'-cyanine iodide (PIC) in the inset. Linear absorption in the monomer (...) and J-aggregate (-) in aqueous solution at a concentration of $2.5 \times 10^{-4}$ and $2.0 \times 10^{-3} \mathrm{M}$, respectively. Measurements were done using a UV-Vis-NIR CARY-500 SCAN spectrophotometer from 200 to $2000 \mathrm{~nm}$.

at concentrations of $2.5 \times 10^{-4}$ and $2.0 \times 10^{-3} \mathrm{M}$, respectively. These measurements were done using a UV-vis-NIR CARY-500 SCAN spectrophotometer. The J-aggregate preparation is well described in Ref. 13. Briefly, PIC monomer from Acros Organics, used without further purification, was dissolved in DI water to a concentration of $2.5 \times 10^{-4}$ or $2.0 \times 10^{-3} \mathrm{M}$. Then, $0.2 \mathrm{M} \mathrm{NaCl}$ was added to the more concentrated solution and heated at $60{ }^{\circ} \mathrm{C}$ for $30 \mathrm{~min}$ to form the J-aggregate. The formation of the J-aggregate was corroborated by the presence the strong $J$-band at $573 \mathrm{~nm}$ (see Fig. 1). An interesting feature in this spectrum is the absence of linear absorption at wavelengths greater than ca. $600 \mathrm{~nm}$. This indicates that excitation beyond $600 \mathrm{~nm}$ can only occur through nonlinear (multiphoton) processes. In addition, since the $J$-band corresponds to an enhanced one-photon allowed transition, it should also be allowed for three-photon excitation. Thus, a strong 3PA in the supramolecular assembly is predicted. Because there is no significant linear absorption in the monomer solution at $573 \mathrm{~nm}$, we should not expect any significant 3PA in this solution at the equivalent energy.

The measurements of the 3PA coefficient of PIC monomer and J-aggregate in aqueous solution at concentrations of $2.5 \times 10^{-4}$ and $2.0 \times 10^{-3} \mathrm{M}$, respectively, were done at $1720 \mathrm{~nm}(3 \times 573 \mathrm{~nm})$ using the well known open aperture $Z$-scan technique. ${ }^{18}$ The PIC solutions were placed in a $0.1 \mathrm{~mm}$ path length cell and pumped with a tunable OPG pumped by the third harmonic of a mode-locked, 25 ps fullwidth at half-maximum (FWHM) Nd-YAG laser (EKSPLA), operating at $10 \mathrm{~Hz}$ repetition rate. The beam waist at the focal plane was ca. $28 \mu \mathrm{m}$. Very low irradiances of approximately $0.2 \mathrm{GW} / \mathrm{cm}^{2}$ were required for the 3 PA measurements in the J-aggregate. We should highlight that the employed irradiances were below the photon fluence for irreversible changes in the J-aggregate optical properties. ${ }^{17}$ According to anisotropy studies of PIC-J-aggregates reported in the literature, ${ }^{13}$ the induced transition at $573 \mathrm{~nm}$ corre-

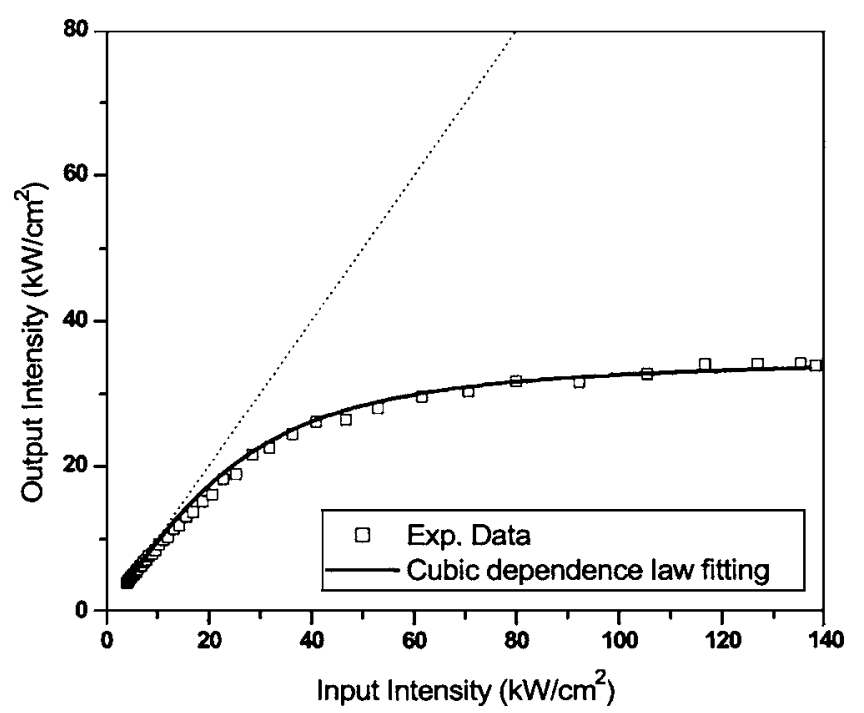

FIG. 2. Transmitted intensity vs incident intensity of PIC J-aggregate/ $\mathrm{H}_{2} \mathrm{O}$ solution at $2.0 \times 10^{-3} \mathrm{M}(\square)$. The solid line represents the theoretical best-fit curve, using 3PA equation with the parameter $\alpha_{3}=(2.3 \pm 0.3)$ $\times 10^{-15} \mathrm{~cm}^{3} \mathrm{~W}^{-2}$. The dashed line corresponds to the data for pure water.

sponds to the lowest excitonic state $S_{1}$ with a transition dipole moment orientation nearly parallel to the molecular chain direction.

Pure three-photon absorption in PIC J-aggregate was corroborated by the transmitted intensity versus incident intensity plot (Fig. 2). The cubic dependence law obtained for the J-aggregate $/ \mathrm{H}_{2} \mathrm{O}$ solution at a concentration 2.0 $\times 10^{-3} \mathrm{M}$ confirmed 3PA for PIC J-aggregate at $1720 \mathrm{~nm}$. The solid line is the theoretical fitting using a cubic dependence law.

In Fig. 3, the normalized measured transmittance versus sample position $Z$ is shown, pumping at $1720 \mathrm{~nm}$ for the J-aggregate, at three different energies. The solid lines are

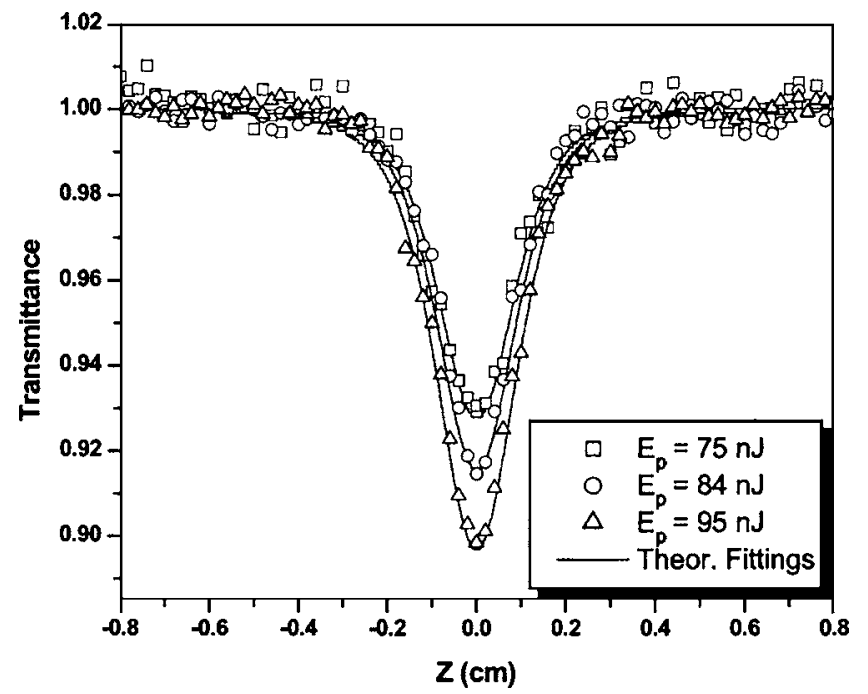

FIG. 3. Open aperture Z-scan curves of PIC J-aggregate taken at three different pump energies (symbols) and corresponding theoretical fittings for 3PA (-). Measurements were done using in $0.1 \mathrm{~mm}$ path cell using a tunable OPG pumped by the third harmonic of a Mode-Locked, $25 \mathrm{ps}$ fullwidth at half-maximum (FWHM), Nd-YAG laser (EKSPLA), operating at a $10 \mathrm{~Hz}$ repetition rate. The beam waist at the focal plane was ca. $28 \mu \mathrm{m}$ at $\lambda_{p}=1720 \mathrm{~nm}$. 
the corresponding theoretical fittings using basic theoretical considerations for three-photon absorption. Employing the same equation derived by Hernandez and co-workers. ${ }^{8}$ to obtain the normalized energy transmittance for pulses with temporal and spatial Gaussian profile, and assuming negligible linear absorption at the pump wavelength, the average value of the 3PA coefficient $\alpha_{3}=(2.3 \pm 0.3) \times 10^{-15} \mathrm{~cm}^{3} \mathrm{~W}^{-2}$ was obtained. Then, relating $\alpha_{3}$ with the PIC concentration $d_{0}(\mathrm{M})$ in the J-aggregate and the photon energy $[h(c / \lambda)]^{8}$, this coefficient yielded a 3PA cross-section $\sigma_{3}^{\prime}=2.5 \pm 0.4$ $\times 10^{-71} \mathrm{~cm}^{6} \mathrm{~s}^{2}$ photon $^{-2}$ for the supramolecular assembly. This extraordinary $\sigma_{3}^{\prime}$ value can be explained by understanding better the parameters that determine the 3PA crosssection of organic molecules.

According to Luo and co-workers, theoretical calculations for 3PA in organic molecules using truncated sum-overstates (SOS), ${ }^{19}$ the only parameter that contributes to the final 3PA cross-section is the transition dipole moment between the ground state and the final state, $\mu^{0 f}$. On the other hand, for a large number of identical PIC molecules with parallel dipole moments, the electronic transitions of individual molecules are strongly coupled by their excitonic interaction, resulting in an enormous transition dipole moment. Therefore, the resulting large transition dipole moment in the J-aggregate is, consequently, responsible for the unprecedented high $\sigma_{3}^{\prime}$ value found in the PIC supramolecular assembly. This strong coupling was also observed in aggregates by the presence of a phenomenon called superirradiance. $^{14,20,21}$

We also thought about the role excimer formation could play in such a big enhancement. It is well known that an excimer is a pair of identical planar molecules arranged face to face, and which interaction is repulsive in the ground state but attractive if at least one of the molecules is in the excited state. If the intermolecular distance is less than $4_{A}^{0}$ the $\pi$-orbitals of both molecules can interact, allowing an extended electron delocalization over more than one molecule. The presence of this effect is usually manifested by the presence of a redshifted emission band in the aggregate. Because we did not see any evidence of excimer formation from the absorption and fluorescence spectra, we consider its contribution to be negligible. ${ }^{13}$

Similar measurements were performed in a monomer solution at concentration $2.5 \times 10^{-4} \mathrm{M}$ and much higher irradiances $\left(\sim 10 \mathrm{GW} / \mathrm{cm}^{2}\right)$, and no measurable 3PA was observed for the monomer solution.

\section{CONCLUSIONS}

We have demonstrated an unprecedented 3PA crosssection enhancement in a supramolecular J-aggregate assembly. The origin of such a high value is the strong transition dipole moment in the supramolecular assembly. No experimental evidence of pure 3PA in the monomer was observed. This encouraging result will pave a new road for the design of new multiphoton absorbing organic materials through supramolecular assembly.

\section{ACKNOWLEDGMENTS}

This research was partially supported by start up funds provided to FEH by the Department of Chemistry, University of Central Florida. We wish to acknowledge the Civilian Research and Development Foundation (UK-C2-2574-MO-04), the donors of The Petroleum Research Fund of the American Chemical Society, and the National Science Foundation (Grant No. ECS-0217932 and Grant No. DMR-9975773) for partial support of this work.

${ }^{1}$ Winfried Denk, James H. Strickler, and Watt W. Webb, Science 248, 73 (1990).

${ }^{2}$ Ion Cohanoschi, Kevin D. Belfield, and Florencio E. Hernández, Chem. Phys. Lett. 406, 462 (2005).

${ }^{3}$ E. M. Attas, M. G. Sowa, T. B. Posthumus, B. J. Schattka, H. H. Mantsch, and S. L. L. Zhang, Biopolymers 67, 96 (2002).

${ }^{4}$ Nonlinear Optics, edited by R. W. Boyd (Academic, San Diego, CA, 1992), Chaps. 4 and 8.

${ }^{5}$ Min Gun, Opt. Lett. 21, 988 (1996).

${ }^{6}$ S. Singh and L. T. Bradley, Phys. Rev. Lett. 12, 612 (1964).

${ }^{7}$ W. M. McClain, R. A. Harris, and E. C. Lim (Academic, New York), p. 1 (1977).

${ }^{8}$ Florencio E. Hernandez, Kevin D. Belfield, and Ion Cohanoschi, Chem. Phys. Lett. 391, 22 (2004).

${ }^{9}$ Ion Cohanoschi and Florencio E. Hernández, J. Phys. Chem. B (in press).

${ }^{10}$ E. E. Jelley, Nature 138, 1009 (1936).

${ }^{11}$ G. Scheibe, Angew. Chem. 49, 563 (1936).

${ }^{12}$ E. Collini, C. Ferrante, and R. Bozio, J. Phys. Chem. B 109, 2 (2005).

${ }^{13}$ K. D. Belfield, M. Bondar, F. E. Hernandez, O. Przkonska, and Sheng Yao, Chem. Phys. (in press).

${ }^{14}$ F. C. Span and S. Mukamel, J. Chem. Phys. 91, 683 (1989).

${ }^{15}$ H. Fidder, J. Knoester, and D. A. Wiersma, J. Chem. Phys. 98, 6564 (1993).

${ }^{16}$ J. Rosker, H. O. Marcy, T. Y. Chang, J. T. Khoury, K. Hansen, and R. L. Whetten, Chem. Phys. Lett. 196, 427 (1992).

${ }^{17}$ R. Gardonas, A. Pugzlys, and A. Piskarskas, Exp. Tech. Phys. (Lemgo, Ger.) 41, 57 (1995).

${ }^{18}$ M. Sheik-Bahae, A. A. Said, T. Wei, D. J. Hagan, and E. W. Van Stryland, IEEE J. Quantum Electron. 26, 760 (1990).

${ }^{19}$ P. Cronstrand, P. Norman, Y. Luo, and H. J. Agren, J. Chem. Phys. 121, 2020 (2004).

${ }^{20}$ J. Feldman, G. Petre, E. O. Gobel, P. Dawson, K. Moore, C. Foxon, and R. J. Elliot, Phys. Rev. Lett. 59, 2337 (1987).

${ }^{21}$ S. de Boer and D. A. Wiersman, Chem. Phys. Lett. 165, 45 (1990). 